\title{
Studies of Elastic and Unelastic Properties of Ferroelectrics under High Uniaxial Stress
}

\author{
Valery M. Tsaplev \\ Department of Physics, North-West Polytechnical Institute, Millyonnaya St., 5, St. Petersburg, 191186, RUSSIA
}

\begin{abstract}
Ferroelectric ceramics are widely used in various acoustical applications as active materials for different transducers. These transducers often operate under the influence of high mechanical stresses, both hydrostatic and uniaxial. Uniaxial stresses significantly change the properties of ferroelectrics.

The study of the uniaxial stress dependence of the elastic moduli and internal friction is, therefore, important for two reasons. First - from a practical point of view, for the purposes of designing transducers to operate under such conditions. Second - the elastic modulus and internal friction of the material depend on its internal physical structure (domain structure, point defects, dislocations, etc.). Consequently, acoustical methods of studying these properties, being highly precise, can provide much very useful information from a physical point of view. While the dependence of the dielectric properties on uniaxial stress have been studied by a great number of investigators, information on the dependence of the elastic modulus and internal friction on stress is very limited, though such studies began long ago. Mostly the lack of such investigations is accounted by the difficulties in making measurements. In this paper the resonance method of measuring the internal friction and elastic modulus under the influence of rather high uniaxial stresses is described. The method and apparatus were designed by the author long ago, but since then they have been improved significantly and new results have been obtained that have not been published before. This paper is concerned with only the results obtained by the resonance method and is not concerned with the results obtained by the pulse-interference method that was used in the $\mathrm{MHz}$-frequency range.
\end{abstract}

\section{INTRODUCTION}

Studies of the elastic and non-elastic properties of uniaxially pre-stressed solid bodies can provide much very useful information about the internal physical properties. These properties have been under investigation by many scientists. Most of these studied the static modulus, though dynamic moduli can be measured much more accurately and can provide much more information.

Attempts at using accurate ultrasonic methods (especially in the kilohertz frequency range, where it is necessary to use the resonance technique) under high stress usually are not successful, mostly because of two conflicting demands, both of which must be satisfied. The sample must be "acoustically free" ( that means, that it can vibrate freely, without any significant losses of energy through the suspension or supporting system ). At the same time the supporting system must be strong enough to transfer very high mechanical forces.

These limitations are not so severe in the MHz-frequency range, where the pulse-interference method can be used, although still with many specific difficulties. The method and apparatus used in this frequency range, as well as the results obtained are not in the scope of this paper. One can find some of the results in reference ${ }^{1}$, and more recent results are the subject of a later paper.

As for frequency range $30-100 \mathrm{kHz}$, as far as the author knows, one of the first attempts of such measurements, was made by Whymark and Witting ${ }^{2}$. They used specially designed specimens mounted to measure the displacement at the resonance, the mechanical $Q$-factor and the relative coupling factor of some piezomagnetic materials under high uniaxial pre-stress. A significant influence was found. Obviously, the high uniaxial stress somehow affect the internal structure of the material. Since the ultrasonic method is very accurate, it is excellent for studying this influence.

\section{STUDIES OF FERROELECTRICAL CERAMICS}

\subsection{Short Theoretical Background}

The idea of using ultrasonic methods to study the elastic properties and the internal friction of uniaxially pre-stressed ferroceramics was first used because it was practical. The ferroceramics are used in deep sea sonars and the material changes its properties under steady stress. Moreover, this change is continuous one and goes on for hours and even days after the stress is applied. So it was necessary to know something about the behaviour of the elastic modulus of the pre-stressed material. But later, in the course of the experiments, the scientific goals became the most important and we tried to determine the physical mechanisms responsible for the behaviour of ceramic materials under stress.

Many investigators have studied the different properties of ferroceramics under mechanical stress ${ }^{3}$ and that the stressstrain dependence is essentially non-linear. Non-linearity becomes significant for stresses above $20 \mathrm{MPa}$ with elastic hysteresis. Strain increases under stress that is maintained for 